Cahiers $d u$ MONDE RUSSE

\section{Cahiers du monde russe}

Russie - Empire russe - Union soviétique et États indépendants

$46 / 4 \mid 2005$

L'invention d'une politique humanitaire

\title{
Le soutien du zemgor aux écoles de la diaspora
}

\section{Lidija I.PETRUŠEVA}

\section{OpenEdition \\ Journals}

Édition électronique

URL : https://journals.openedition.org/monderusse/9438

DOI : $10.4000 /$ monderusse. 9438

ISSN : $1777-5388$

Éditeur

Éditions de l'EHESS

Édition imprimée

Date de publication : 1 décembre 2005

Pagination : 831-843

ISBN : 2-7132-2057-2

ISSN : $1252-6576$

\section{Référence électronique}

Lidija I.PETRUŠEVA, « Le soutien du zemgor aux écoles de la diaspora », Cahiers du monde russe [En ligne], 46/4 | 2005, mis en ligne le 01 janvier 2005, consulté le 03 septembre 2022. URL : http:// journals.openedition.org/monderusse/9438; DOl : https://doi.org/10.4000/monderusse. 9438 


\section{LE SOUTIEN DU ZEMGOR AUX ÉCOLES DE LA DIASPORA}

L'histoire de l'assistance aux réfugiés a été marquée par la création de nombreuses organisations sociales dans les différents pays d'accueil. Fondées par les émigrés, ces organisations comprenaient notamment le Comité des zemstvos et des villes d'aide aux citoyens russes à l'étranger et ses représentations locales. Ce comité, avec le Zemgor de Prague, a occupé une place singulière. L'investissement de cette organisation, dont l'action a touché des domaines très variés - de la création de cantines gratuites à l'organisation des migrations de réfugiés - , a été primordial dans le développement de la vie éducative de l'émigration.

Dès 1921, le Comité avait défini parmi ses objectifs prioritaires la création d'écoles :

le souci d'éduquer et de former les enfants - qui, déjà dans des conditions normales d'existence, est l'un des devoirs essentiels de l'État et de la société revêt une signification exceptionnelle dans ce moment difficile, que traverse la Russie, d'effondrement de l'économie et des structures étatiques ${ }^{1}$.

L'éducation fut ainsi d'emblée présentée comme une priorité dans l'activité de l'émigration. La situation des plus jeunes, dont l'enfance avait été marquée par l'épreuve de la guerre civile et qui se trouvaient désormais dispersés dans différents pays étrangers, nécessitait une action spécifique. La création d'établissements russes ne répondait pas seulement à une préoccupation d'ordre identitaire, à savoir préserver les enfants du risque de dénationalisation, il s'agissait de recréer un environnement familier qui permette de panser leurs traumatismes.

La plupart des écoles et des lycées russes créés au cours des années 1920 enseignaient les disciplines classiques russes, mais également de nouvelles matières et des programmes proposés dans les établissements scolaires européens, de façon à

1. GARF [Gosudarstvennyj Arhiv Rossiskoj Federacii - Archives d'État de la Fédération de Russie],f. R 5809, op. 1, d. 185,1. 88. 
donner aux élèves les moyens de poursuivre leur formation supérieure à l'étranger. Mais ces écoles, avant tout conçues pour éduquer les enfants dans leur culture d'origine, furent des lieux privilégiés de transmission de l'héritage identitaire russe. Comme le faisait remarquer le pédagogue A. A. Dehtjarev :

ce n'est qu'à l'école que les enfants commencent à connaître la Russie, car c'est là que brûle, comme le buisson ardent, l'essence de la Russie, c'est là que l'on parle constamment de la Russie, et en classe, et dans les dortoirs. L'identité russe et le sentiment d'amour de la Russie deviennent des réalités claires et concrètes de l'existence quotidienne ${ }^{2}$.

Le nombre d'enfants dans l'émigration varie beaucoup selon les sources et les estimations. Les données établies par le Zemgor de Paris en 1921 faisaient état de 19526 enfants (20500 d'après la Croix-Rouge russe, dont 10847 dans les Balkans, 6096 en Finlande, 2420 en Estonie et 1128 en Afrique) ${ }^{3}$. Les pays Baltes, la Pologne, la Turquie, la Bulgarie, la Tchécoslovaquie, la Yougoslavie, la Grèce, ainsi que les pays d'Europe occidentale comptaient parmi les pays d'accueil disposant d'établissements russes soutenus par le Zemgor de Paris. L'organisation recevait des fonds du Conseil des ambassadeurs, des gouvernements des États slaves, ainsi que d'un certain nombre d'organisations humanitaires étrangères. Le Zemgor soutenait directement 45 des 90 écoles de l'émigration, soit 5500 enfants ${ }^{4}$.

Le Zemgor et les organisations pédagogiques avaient des objectifs éducatifs communs, mais dans chacun des pays leur activité était conditionnée autant par la situation des groupes de réfugiés que par les liens établis avec les autorités et les sociétés des pays d'accueil.

En Yougoslavie, Bulgarie et Tchécoslovaquie, les gouvernements manifestèrent un soutien important aux établissements scolaires russes. Dans les États issus du démantèlement de l'ancien empire russe (Finlande, pays Baltes et Pologne), le nombre des anciennes écoles impériales chuta spectaculairement malgré la présence d'importantes minorités russes. Dans les autres pays d'Europe centrale et occidentale, qui étaient soit des pays d'accueil très mineurs de l'émigration (Grande-Bretagne, Grèce, Suisse, Autriche, Belgique), soit au contraire des centres importants (France et Allemagne), la gratuité de l'enseignement entraîna la scolarisation de la plupart des enfants dans les établissements nationaux. Dans ces pays, le Zemgor soutint prioritairement les écoles secondaires russes qui étaient en général payantes et dont l'existence était, pour la plupart d'entre elles, redevable à des initiatives privées. Ainsi, en France ou en Allemagne, la proportion d'enfants scolarisés dans les établissements russes était-elle minime par rapport au nombre d'émigrés installés dans ces pays.

2. GARF, f.R 5785 , op. 2, d. 29,1. 3.

3. GARF, f. R 9135, op. 1, d. 9,1.7.

4. GARF, f. R 5785 , op. 2, d. $56,1.1$. 
$\mathrm{Au}$ IIe congrès des organisations pédagogiques tenu à Prague en 1925, V. V. Rudnev, membre du Zemgor de Paris et auteur de L'école russe à l'étranger (Paris, 1924), fit une intervention sur la situation financière et les perspectives des établissements scolaires russes. Il soulignait que seulement $21 \%$ des 16500000 francs du budget consacré à l'éducation provenaient de sources russes. Les finances dévolues aux écoles variaient considérablement selon les pays. Dans les États slaves, $97 \%$ du budget étaient assurés par les États (50\% par la Yougoslavie, $39 \%$ par la Tchécoslovaquie et $8 \%$ par la Bulgarie ${ }^{5}$ ).

L'analyse de la situation et des perspectives de développement de l'éducation russe révélait l'énorme disparité entre le nombre de réfugiés et le nombre d'enfants scolarisés dans les établissements de l'émigration. Dans les États slaves, où l'on dénombrait environ 110000 réfugiés, 5510 enfants étaient pris en charge dans 42 écoles. Dans les États issus de l'empire russe (Finlande, pays Baltes, Pologne, Bessarabie), sur les 220000 réfugiés recensés, seuls 2850 enfants fréquentaient les 31 écoles russes existantes. Quant aux autres pays d'Europe centrale et occidentale et leurs 420000 réfugiés, ils ne disposaient que de 17 écoles qui accueillaient 620 enfants $^{6}$.

Au cours des premières années, le Zemgor de Paris finança un certain nombre d'établissements scolaires à Constantinople : 3 lycées (soit plus de 900 élèves), 3 collèges, 10 écoles élémentaires, 2 internats, 10 jardins d'enfants, 6 cantines. L'aggravation de la situation politique dans la région et les évacuations massives des réfugiés russes de Turquie qui s'ensuivirent incitèrent le Zemgor à transférer les écoles existantes principalement en Bulgarie, en Serbie et en Tchécoslovaquie. Ce dernier pays joua un rôle particulier dans l'éducation des jeunes émigrés par le biais du programme, «L'action russe », mis en œuvre par le gouvernement de Masaryk. Des milliers d'enfants et de jeunes purent bénéficier de ce programme de soutien à l'éducation gratuite russe (primaire, secondaire et supérieure) pris en charge par l'État. Le gouvernement finança intégralement 10 écoles secondaires. L'une d'elles avait été fondée à Constantinople avant d'être réinstallée à Třebova en Moravie. En 1922, ce lycée accueillait plus de 500 enfants et encore 419 au cours de l'année scolaire 1929-1930. L'établissement était géré par le ministère de l'Éducation nationale tchécoslovaque. En septembre 1922, un autre lycée russe fut ouvert à l'initiative du Zemgor de Prague. À ses débuts, il accueillait 16 élèves, 230 en 1924 et 296 en 1929. À partir de 1928, il fut également pris en charge par le ministère. En 1935, les deux lycées de Moravie et de Prague fusionnèrent pour ne plus former qu'un établissement dans la capitale. Dans ce pays, les établissements russes étaient intégrés au réseau éducatif national, ce qui permettait aux enseignants russes d'être assimilés au corps des fonctionnaires. L'État tchécoslovaque contribua, par le biais des représentants du Zemgor tchèque, au financement d'autres écoles russes à l'étranger, notamment en 1923, lorsque les financements du Conseil des ambassadeurs furent subitement réduits.

\footnotetext{
5. GARF, f. 5785, op. 2, d. 18,1. 15-16.

6. GARF, f. 5785 , op. 2 , d. $18,1.18$.
} 
Prague était également le centre de la vie pédagogique russe en émigration. En effet, c'est dans cette capitale que le Bureau pédagogique pour les écoles élémentaires et secondaires russes fut fondé lors du Ir congrès des enseignants de ces écoles en 1923. Ce bureau, qui allait jouer un rôle décisif dans l'organisation et l'harmonisation des programmes d'enseignement, était composé de 19 personnes, dont 12 délégués du congrès et 7 représentants d'organisations sociales de l'émigration. Le comité directeur du Zemgor y était représenté par Z. A. Makšeev, le professeur N. M. Mogiljanskij, V. V. Rudnev, P. P. Jurenev ; le comité central de l'Union des villes par P. D. Dolgorukov. Plusieurs congrès annuels de pédagogues (de 1924 à 1927) et d'étudiants (1921-1922 et 1924) furent organisés à Prague au cours des années 1920. Le Zemgor de Prague publia par ailleurs un organe de liaison, $L$ 'école russe à l'étranger.

La Yougoslavie fut celui des pays d'accueil de l'émigration russe qui prit en charge le plus d'élèves et consacra les plus gros budgets à l'éducation des jeunes émigrés. Selon les données de la commission de soutien aux réfugiés russes, 5317 enfants se trouvaient dans le pays vers 1924, dont 4025 d'âge scolaire7 (soit la moitié des enfants inscrits dans des écoles russes en Europe) sur les 38000 réfugiés enregistrés à cette date dans le royaume ${ }^{8}$. L'État yougoslave finança la majorité des établissements scolaires installés dans le pays, y compris trois écoles de cadets évacuées de Turquie (celles de Crimée, du Don et de Russie) et deux instituts de jeunes filles. La commission de soutien avait à sa charge sept établissements. Le comité directeur du Zemgor fonda quelques-unes des écoles d'enfants qui furent prises en charge localement par le Comité des villes sous la responsabilité de V. D. Brjanskij. La Commission de soutien et le Zemgor finançaient conjointement 14 établissements scolaires.

Les écoles russes de Yougoslavie avaient le statut d'établissements d'État. À la fin des années 1920, certaines étaient toujours en activité (les corps de cadets, les instituts de jeunes filles, 5 lycées) et accueillaient alors 745 élèves 9 .

Le Zemgor de Paris manifesta une attention particulière à la situation des enfants en Bulgarie. Selon les données du Comité, vers 1923 la Bulgarie avait recueilli 34000 réfugiés, dont 2000 enfants en âge d'être scolarisés. La plupart (1 674) étaient pris en charge par les écoles russes, mais 300 d'entre eux se trouvaient hors de tout cadre scolaire ${ }^{10}$. Les moyens financiers de l'école russe en Bulgarie provenaient de fonds très divers : ceux de l'État bulgare, du comité directeur du Zemgor, de la Société des Nations, des gouvernements cosaques du Terek et du Don. La société de bienfaisance du professeur Whittemore attribuait des bourses d'études à des élèves nécessiteux. Certains des établissements avaient été transférés de

7. V. V. Rudnev, Zarubežnaja russkaja škola, 1920-1924, P., 1924, p. 26.

8. Les différentes évaluations du nombre d'émigrés dans les différents pays proviennent des archives du GARF (pour les estimations de l'année 1921 : f. R 9135, op. 1, d. 9, 1. 4 et pour l'année 1924 : f. R 5764, op. 5, d. 1,1.48).

9. GARF, f. R 5785, op. 1, d. 50,1.1-11.

10. GARF, f. R 5809, op. 1, d. 79, 1. 73. 
Turquie ou d'Afrique, tels le lycée de Gallipoli, le lycée V. V. Neratova de Constantinople ou encore le corps des cadets du Don d'Ismaïlia. La Bulgarie contribua à la mise en place de nouvelles écoles primaires et élémentaires. En 1922, il en fut créé 19 avec le statut d'écoles d'État.

Le premier établissement scolaire russe de Bulgarie fut ouvert à Varna : il accueillit de nombreux réfugiés dès avant la fin de la guerre civile. Un lycée, dirigé par S. N. Kononovič (ancien directeur du lycée d'Odessa) ouvrait ses portes dans cette ville en avril 1920. Puis en juin de la même année, c'était à Sofia l'ouverture d'un lycée russe placé sous la responsabilité de l'ancien directeur du lycée $n^{\circ} 3$ d'Odessa, A. P. Stepanov, et administré par le responsable local de l'Union des villes, le général A. V. Arciševskij. D’autres établissements furent également fondés à Chumen, Dol'na Orehovic, Plovdiv et Pechter.

Cependant en 1921-1923, et en dépit des efforts consentis de part et d'autre, le réseau scolaire russe traversa une grave crise financière. La situation économique et politique de la Bulgarie ne permettait plus à son gouvernement de disposer des moyens nécessaires à la prise en charge des écoles ; celle-ci revint presque exclusivement au Zemgor qui éprouva les plus grandes difficultés à l'assumer. Conscients de leur incapacité à assurer les salaires des professeurs et de l'état de malnutrition des enfants, le Comité directeur et l'Union des villes intervinrent à plusieurs reprises à ce sujet auprès du Haut commissariat pour les réfugiés de la Société des Nations.

En juillet 1922, le représentant de la SDN, Collins, rencontra à Sofia les directeurs des écoles russes de Bulgarie et proposa, au cas où la SDN ne pourrait répondre aux urgences financières, que 200 enfants fussent envoyés en France où ils seraient répartis dans des familles de fermiers. Mais les responsables pédagogiques n'envisagèrent cette solution que pour les orphelins et les élèves de plus de 17 ans et demandèrent que d'autres propositions fussent faites pour la majorité restante dont ils craignaient que la dispersion dans des familles françaises n'entraînât une assimilation rapide.

Aucun subside ne vint de la SDN, mais en août 1923, le nouveau gouvernement du professeur Tsankov prit la décision de débloquer des fonds réguliers pour les écoles russes (500 $000 \mathrm{lev}$ ), ce qui permit, mais en partie seulement, de financer leur fonctionnement. Vers 1930, les lycées de Varna, Chumen, Sofia, Pechter ainsi que quelques maisons d'enfants réparties dans différentes villes du pays étaient toujours en activité, rassemblant au total 1085 enfants $^{11}$.

Dans les États issus de l'effondrement de l'empire russe, la situation se présentait différemment: ils avaient dû faire face à deux vagues d'émigration, l'une générée par la guerre civile, la seconde par l'avènement de la Russie soviétique. Les gouvernements de ces pays, peu favorables aux populations russophones, ne permirent pas la création d'écoles d'État et réduisirent même le nombre des écoles russes créées avant la révolution. Le comité du Zemgor adressa plusieurs plaintes à ce sujet à la SDN en s'appuyant sur le droit des minorités nationales nouvellement 
garanti à l'échelle internationale. La situation scolaire ne s'améliora pas pour autant et le réseau d'établissements russes s'effondra rapidement, particulièrement en Bessarabie où, au milieu des années 1920, il n'existait plus que quelques écoles privées russes menacées de fermeture par une administration tatillonne qui exigeait d'elles un renouvellement annuel des inscriptions. Cette situation préoccupante se trouva au centre des débats du II $^{\mathrm{e}}$ congrès pédagogique de Prague qui fut alors conduit à rédiger la résolution suivante : «Face à la population russe sans défense de Bessarabie, le gouvernement roumain agit dans ses actes et ses résolutions comme si les nationaux russes de Bessarabie n'existaient pas $»^{12}$.

La situation n'était guère meilleure en Pologne. En 1924, le pays comptait 60800 réfugiés. Seulement $1 \%$ des enfants était scolarisé dans les écoles russes, pour la plupart des écoles privées ou subventionnées par les organisations sociales de l'émigration. La situation s'améliora au cours de la seconde moitié des années 1920, en particulier grâce au soutien apporté à certains des établissements par des notables locaux. Mais la précarité apparut telle à la fin de la décennie qu'il fut question de créer une «caisse des écoles » pour suppléer l'incapacité financière des parents à fournir aux enfants le matériel scolaire le plus élémentaire. En 1927-1928, selon les données du Bureau pédagogique de Prague, 2362 enfants étaient scolarisés dans les 16 écoles russes en activité à Varsovie, Wilno, Brest, Rovno, Pinsk, Lutsk, Grodno (soit, pour la plupart, dans les régions orientales du pays peuplées de nombreuses minorités) ${ }^{13}$.

La situation était plus favorable dans les pays riverains de la Baltique.

En Finlande, le nombre de réfugiés ne cessa de décroître dans la première moitié des années 1920 passant de 25000 à 14300 en 1924 et les enfants de réfugiés furent pris en charge dans les écoles russes, anciennement impériales. Au milieu de la décennie, la stabilisation des flux de réfugiés incita les paroisses et les organisations sociales russes à prendre quelques initiatives. Le comité du Zemgor fut amené à subventionner dix écoles, six d'enseignement secondaire et quatre d'enseignement élémentaire, soit respectivement $80 \%$ et $55 \%$ d'entre elles. En 1926-1927, le Zemgor soutenait financièrement 14 établissements scolaires réunissant 662 enfants $^{14}$.

En Lettonie, le Comité du Zemgor avait développé son action d'assistance dès 1921 et apportait une aide financière à l'école russe de Riga avec le concours de la Croix-Rouge russe et du Comité des émigrés russes. Il participa à la création du jardin d'enfants de la capitale, d'un orphelinat et de l'école qui lui était rattachée ainsi qu'à la fondation de l'université populaire et contribua à l'enseignement de la langue lettone. À partir de 1924, l'amenuisement des fonds du Zemgor ne lui permit plus de maintenir ces soutiens, l'orphelinat et quelques bourses d'études exceptés.

En Estonie vers 1924, 91000 personnes, soit plus de $8 \%$ de la population du pays, étaient de nationalité russe, 19000 d'entre elles étaient des réfugiés ${ }^{15}$. Le

12. GARF, f. R 5785 , op. 2, d. 46, 1. 238 bis.

13. GARF, f. R 5785 , op. 1, d. 48.

14. GARF, f. R 5785, op. 1, d. 43, 1. 1-23.

15. Rudnev, Zarubežnaja russkaja škola , p. 193. 
comité des émigrés russes, dirigé par V. A. Rogožnikov (représentant du Zemgor), fut à l'origine du développement du réseau scolaire russe financé par le Zemgor parisien. D'après les statistiques du bureau pédagogique, 14 des 20 établissements existants dataient de l'époque impériale. À la fin des années 1920, on dénombrait 2890 enfants scolarisés ${ }^{16}$.

En Lituanie, contrairement à l'Estonie, les écoles russes impériales avaient toutes disparu au cours de la Première Guerre mondiale en raison de l'occupation de la région par les armées des puissances centrales et de l'évacuation de la majorité de la population civile en 1915. Le recensement lituanien de 1923 fait état de la présence de 55000 Russes. Le premier lycée russe de Kaunas (anciennement Kovno) fut créé en 1920 et placé sous la tutelle du ministère de l'Éducation nationale. En 1925-1926, ce lycée payant accueillait 238 élèves, dont 62 étaient des enfants d'émigrés russes. Par la suite, des cours pour adultes y furent dispensés, d'autres encore étaient destinés aux classes élémentaires. Un orphelinat fut également ouvert dans la capitale. En 1926-1927, l'effectif scolaire des établissements russes du pays s'élevait à 1000 élèves ${ }^{17}$.

Dans certains pays d'Europe centrale et du sud-est, très marginaux dans l'histoire de la diaspora, de réels efforts furent déployés au profit de l'éducation des enfants russes. Ainsi, en Hongrie (pays qui comptait 5020 Russes en 1921), à l'initiative du Comité russe en Hongrie et de la branche locale de la Croix-Rouge, les fonds du Zemgor permirent la création d'une école élémentaire.

En 1926, la Grèce comptait 3040 Russes, dont 152 enfants d'âge scolaire : 70 d'entre eux fréquentaient les écoles russes et 39 des établissements grecs ${ }^{18}$. En mars 1921, le soutien du Zemgor avait permis l'ouverture d'un lycée russe à Athènes qui avait accueilli 54 enfants cette année-là (40 en 1929) ${ }^{19}$. Comme toutes les institutions éducatives russes, le lycée d'Athènes souffrait d'un manque chronique de moyens et complétait son budget en organisant chaque année un arbre de Noël. Sa situation financière s'aggrava à l'aube de la nouvelle décennie lorsque le Zemgor fut amené à pratiquement cesser son aide (lui-même privé des fonds qu'il recevait jusqu'alors des États-Unis). La direction de l'établissement sollicita les organisations internationales. L'office Nansen répondit à sa demande en promettant de fournir 46500 drachmes ${ }^{20}$. Le lycée russe d'Athènes, malgré sa carence en moyens, fonctionna jusqu' au milieu des années 1930.

En Europe occidentale, l'Allemagne et la France constituaient les pôles d'accueil les plus importants de l'émigration. En Allemagne, la présence d'abord massive des réfugiés (évaluée à 300000 personnes), ne cessa de décroître avec la détérioration de la situation économique des années 1923-1924, au profit notamment de la France. Mais ni en Allemagne, ni en France, la capacité d'accueil des

16. GARF, f. R 5785, op. 1, d. 44, 1. 1-25.

17. GARF, f. R 5785 , op. 1, d. 41, 1. 1-6.

18. GARF, f. R 5780, op. 1, d. 11,1. 165.

19. GARF, f. R 5780, op. 1, d. 11,1. 166.

20. GARF, f. R 5781, op. 1, d. 4,1. 35. 
écoles russes ne répondait à la demande. Pratiquement tous les fonds russes et étrangers dédiés à l'éducation avaient été distribués dans les premières années de l'exil aux établissements russes des Balkans et des pays limitrophes de la Russie. En Allemagne et en France, la très grande majorité des enfants de réfugiés fut scolarisée au sein des écoles publiques gratuites.

Les données statistiques relatives à l'Allemagne ont toujours été incomplètes (elles ne fournissent aucun chiffre sur le nombre d'émigrés en Bavière et dans les régions orientales $)^{21}$. En décembre 1920, le pasteur Mazing fut à l'origine de la création du lycée privé Saint-Georges de Berlin. Il accueillait alors 140 élèves et dispensait un enseignement bilingue, en russe et en allemand. Il comptait encore une centaine d'élèves à la fin des années $1920^{22}$. Un nouvel établissement, géré par le groupe académique russe avec le soutien financier du Zemgor, vit le jour en 1921. En outre, plusieurs écoles furent ouvertes, notamment dans les anciens camps de prisonniers de guerre, grâce aux subsides fournis par le Zemgor à son représentant local F. V. Šlippe. En dehors des fonds attribués au fonctionnement des établissements, le comité directeur du Zemgor attribua des bourses aux enfants les plus démunis scolarisés dans les écoles allemandes, consacrant à ce soutien jusqu'à 70000 marks par mois ${ }^{23}$.

L'évaluation de la présence russe en France fut très diverse selon les sources. Le Zemgor estima à 200000 le nombre de réfugiés (le recensement français de 1926 en comptabilisa 65 000, mais à la tribune de la SDN en 1924, les autorités françaises avaient affirmé en avoir accueilli 400 000). Le gouvernement, qui avait pris en charge l'accueil des réfugiés russes à Constantinople, débloqua de nouveaux fonds pour l'éducation des enfants de réfugiés arrivés en France. Il n'y avait pas de centralisation administrative des écoles russes. La Commission franco-russe créée au sein de l'Institut des études slaves pour la distribution des bourses aux étudiants russes fonda une sous-commission pour les établissements scolaires russes animée par deux Russes et un professeur français, Jules Patouillet. Au cours de ses huit années d'activité au sein de la sous-commission, ce dernier favorisa en particulier la mise en place de sections russes dans les lycées français. Le professeur Jules Legras, spécialiste d'histoire et de littérature russe succéda à J. Patouillet en 1929.

Mais pour l'essentiel, l'éducation des enfants russes fut assurée par les émigrés eux-mêmes. « Nous nous efforçons d'aider la jeunesse sans l'aide du gouvernement, sans le soutien d'un quelconque pouvoir, seulement par notre volonté et nos forces sociales » proclamaient les émigrés dans la presse ${ }^{24}$. Mais comme le faisait remarquer V. V. Rudnev lors du congrès des pédagogues russes en mai 1929 , seulement 1500 des 16000 à 20000 enfants que comptait la population russe

21. En 1921, le Zemgor fait état de 512 enfants dont 343 en âge d'être scolarisés (410 selon d'autres données). Voir GARF, f. R 6006, op. 1, d. 11,1. 109.

22. GARF, f. R 5785, op. 1, d. 42,1.2.

23. GARF, f. R 6006, op. 1, d. 11,1. 117 bis.

24. « Naša Smena », Poslednie novosti, 3 mars 1929. 
installée en France (de 150000 à 200000 personnes), étaient scolarisés dans des établissements russes ${ }^{25}$.

En France, la contribution du Zemgor à l'éducation consista surtout en dotations de bourses pour les études et en subsides pour le matériel scolaire. Au cours de la seconde moitié des années 1920, face à l'amenuisement des fonds, le Comité directeur parisien mit en place le système du «vendredi jeûné » (les émigrés étaient invités à se priver de repas ce jour-là et à en reverser le prix dans une caisse créée à cet effet). Cette initiative fut soutenue par de nombreux écrivains, politiciens et hommes de lettres (P. Miljukov, A. Remizov, etc.), les dons furent nombreux et spontanés, notamment ceux des sociétés cinématographiques «Mappemondefilm » et la «Société générale de film » qui, après la sortie de «Volga...Volga » du réalisateur V. Turžanskij, versa 3120 francs à la caisse du « vendredi jeûné » ${ }^{26}$. La France était en effet l'un des rares pays où le Zemgor pouvait trouver des sources de financement dans l'émigration même. Les aides à l'éducation atteignirent 250000 à 300000 francs par an ${ }^{27}$.

L'école secondaire de Paris était l'une des plus anciennes de l'émigration. Fondée en février 1920 par le consulat russe de Paris, à l'initiative d'un groupe de pédagogues, elle bénéficia du soutien de l'ambassadeur V. Maklakov et de sa sœur. L'école fut d'abord placée sous la tutelle du Comité d'œuvre de guerre puis sous celle d'une Société d'aide à l'éducation des enfants réfugiés, présidée par V. Maklakov, assisté de E. P. Kovalevskij. Le directeur de l'école, V. P. Nedačin, était l'ancien directeur du lycée Medvednikovsk de Moscou. Cette école comptait 30 élèves en 1920, 182 en 1929 et en a accueilli 600 au cours de la décennie. Elle était financée par de nombreuses organisations émigrées. Le Zemgor parisien lui versa 336000 francs de subsides entre 1922 et 1929. Le gouvernement français et la municipalité parisienne contribuèrent à son fonctionnement à hauteur, respectivement de 25000 francs et 3000 francs par an. Lorsque les finances de cet établissement atteignirent un point critique, de nombreux représentants de l'émigration se mobilisèrent. La grande princesse Marie Pavlovna fit don de 50000 francs, la ballerine A. P. Pavlova organisa en sa faveur une soirée de bienfaisance qui rapporta 26850 francs. En 1927, L. P. Deterding intervint de façon décisive en permettant l'achat d'une propriété près de Paris, où l'école fut transférée en 1929. L'école prit alors le nom de sa bienfaitrice et resta en fonction jusqu'en $1961^{28}$.

Parmi les premiers établissements apparus dans l'émigration figure également l’École de la marine de Bizerte en Tunisie créée par les corps de marine de Sébastopol et de Vladivostok qui avaient été évacués en 1920 via la Turquie. En 1921, cette école soutenue financièrement par le gouvernement français, et dans une moindre mesure par le Zemgor, accueillit 350 élèves ${ }^{29}$.

25. GARF, f. R 5785 , op. 2, d. $46,1.42$.

26. GARF, f. R 5785 , op. 2, d. 73,1. 76 .

27. GARF, f. R 6094, op. 1, d. 27, 1. 20.

28. GARF, f. R 5913, op. 1, d. 965,1.3.

29. GARF, f. R 5912, op. 1, d. 214, 1. 12. 
Plusieurs des écoles russes de France étaient issues de patronages soutenus par l'aristocratie émigrée, en particulier sur la côte d'Azur où celle-ci s'était établie massivement. L'école russe «Alexandrino» de Nice existait ainsi grâce aux subsides du comité de tutelle du grand prince Andrej Vladimirovič ; l'orphelinat de Cannes, l'orphelinat des jeunes filles étaient sous la protection de la grande princesse Elena Vladimirovna. Des écoles élémentaires virent le jour à l'initiative de paroisses (Paris, La Chalette, Belfort, Ugine, Nilvange, Le Creuset, Billancourt). Plusieurs sections de russes furent créées dans les lycées français.

Dans son rapport sur la situation des écoles secondaires en France durant les années 1920-1925, E. P. Kovalevskij mentionna bien d'autres établissements comme le pensionnat de la famille Rjabušinskij créé en 1921 à Ariane, près de Nice, le foyer pour enfants russes A. Pavlova créé en 1922 à Saint-Cloud et dont les frais de fonctionnement étaient assurés grâce aux fonds personnels d'Anna Pavlova ${ }^{30}$.

Le comité directeur du Zemgor concentra également ses moyens sur l'aide à la formation supérieure en France. Ainsi, à partir de 1924, l'université populaire russe de Paris (créée en 1921) fut placée sous l'administration du Zemgor qui affecta près de 160000 francs à son fonctionnement en 1925-1926 ${ }^{31}$. À cette même époque, 1467 personnes fréquentaient ses différents cours. En outre, le comité parisien apporta une aide substantielle à l'Institut commercial russe de Paris créé en 1925 et dont les diplômes étaient officiellement reconnus par l'État français. Cet établissement, dirigé par V.F. Sollogoub, comptait 65 étudiants en 192932. Un bilan effectué au début de cette année-là faisait état de 41 établissements russes en France $^{33}$.

\section{La crise}

À la fin des années 1920 et au début des années 1930, l'amoindrissement brutal des ressources financières de l'assistance marqua le début de la lutte pour la survie, ainsi qu'en témoigne l'évolution des écoles russes dans la majorité des pays de la diaspora. En 1932, la collecte du «vendredi jeûné » ne rapporta que 30000 francs, contre plus de 100000 l'année précédente ${ }^{34}$. Cette même année 1932, le comité directeur du Zemgor informa la Société des Nations de la situation critique de nombreux établissements, en particulier de l'orphelinat de Montmorency (50 enfants) et de l'université populaire. Il sollicita également une aide pour 175 enfants de chômeurs, des bourses d'études pour 150 personnes en cours de formation professionnelle. En Allemagne, un soutien était demandé pour la cantine des

30. GARF, f. R 5912, op. 1, d. 214,1. 10.

31. GARF, f. R 5785, op. 1, d. 70,1. 157.

32. D'après le bulletin du Zemgor $\left(n^{\circ} 59,1929\right) 269$ adultes y préparaient encore leur baccalauréat en 1929. GARF, f. 5785 , op. 2, d. 50,1.31.

33. Vozroždenie, 6 janvier 1929.

34. GARF, f. R 6094, op. 1, d. 27,1. 87. 
enfants de Berlin (80 élèves). En Estonie, l'urgence était de sauvegarder le lycée russe de Narva (200 élèves), le jardin d'enfants (70 enfants), l'orphelinat (30 enfants), l'hôpital pour enfants. En Pologne, les besoins étaient surtout pressants pour le pensionnat russe de Wilno (30 élèves), en Lettonie, pour le pensionnat russe de Riga (28 enfants). Le Zemgor demanda 50000 francs pour assurer le fonctionnement de l'année scolaire 1932-1933, il en reçut $32650^{35}$.

\section{Corps enseignant et orientation pédagogique}

Jusqu'ici, il a été peu fait mention du corps enseignant, très mal rémunéré, mais qui se dépensa sans compter au service de l'éducation. Selon les propos de l'un des pédagogues, cette activité était le plus souvent considérée comme une «tâche nationale » (služenje nacional'nomu russkomu delu) ${ }^{36}$. Outre l'enseignement proprement dit, il convient de rappeler la contribution d'éminents professeurs à l'encadrement du réseau scolaire à travers l'animation de comités et unions ad hoc, ainsi que leur rôle dans l'élaboration de nouveaux manuels par la suite diffusés dans l'ensemble des pays de la diaspora ${ }^{37}$. Les travaux collectifs effectués lors des différents congrès de pédagogues se concentrèrent en priorité sur les moyens de contrer la dénationalisation des enfants. Comme le soulignait le premier bulletin du lycée russe de Prague, «bien que nous soyons à l'étranger, nous sommes tous les enfants d'une seule patrie $»^{38}$. Le contenu de cette éducation «nationale » fut l'objet des travaux de la conférence de 1924 à laquelle participèrent, entre autres, V. V. Rudnev, les professeurs D. M. Sokol'cev, A. P. Anciferov, P. E. Butenko, V. A. Mjakotin, D. M. Odinec et la comtesse Panin. Cette conférence souligna la nécessité de renforcer l'enseignement des disciplines russes, d'organiser des camps de vacances l'été et, de manière plus générale, des activités culturelles parascolaires. Ces travaux furent poursuivis lors des congrès de 1927 et 1928.

Dans le cours des années 1920, la nouvelle génération d'enfants nés en exil ne connaissait rien de la Russie. Le responsable du lycée de Chumen, A. A. Dehtjarev, avait mené une enquête auprès de 40 élèves nés en Bulgarie. À la question de savoir ce qu'ils connaissaient de la Russie, l'un d'entre eux répondit : " on m'a raconté des choses sur la Russie, mais j'ai oublié » ${ }^{39}$. Bien que de nombreux parents aient tôt perdu tout espoir de retour dans la patrie, ils restaient convaincus de la nécessité d'éduquer leurs enfants dans leur culture d'origine. Mais avec la réduction des

35. GARF, f. R 6094, op. 1, d. 27, 1. 26.

36. GARF, f. R 5785, op. 1, d. 30,1. 34 .

37. Parmi les pédagogues les plus impliqués dans cette tâche, il convient de citer les professeurs D. M. Sokol'cev, I. M. Malinin, V.D. Pletnev, N. M. Mogiljanskij, V. V.Zen’́kovskij, I. A. Bazanov, E. P. Kovalevskij, G. V. Vernadskij et A. L. Bem.

38. GARF, f.R 5809 , op. 1, d. 235, 1. 1.

39. GARF, f. R 5785, op. 2, d. 29, 1. 1. 
ressources et la fermeture progressive des écoles, répondre à cette demande supposait de nouvelles initiatives, de nouvelles méthodes.

C'est ainsi que furent conçues la Journée de la culture russe et la Journée de l'enfant russe, nouvelles fêtes communes à toute l'émigration qui eurent un impact indéniable sur la jeunesse. Le comité du Zemgor prit une part active à l'organisation de ces fêtes annuelles en collaboration avec les enseignants. La première célébration de la Journée de la Culture russe se déroula à Prague en 1925, à l'initiative du Zemgor de Prague et de l'ensemble des organisations académiques et pédagogiques. Par la suite, cette manifestation allait se dérouler dans de nombreux pays de la diaspora. Les Journées de la culture russe firent l'objet de manifestations particulières dans les établissements scolaires de l'émigration. Les responsables du lycée de Třebova en Moravie le soulignaient, « la journée de la culture russe est une fête où l'école occupe le premier rang, en tant que garant de la sauvegarde et du rayonnement de la culture $»^{40}$.

L’idée de promouvoir la Journée de l'enfant russe apparut également à Prague :

« si chaque Russe, qu'il ait ou non des enfants, ne se sent pas responsable du destin de tous les enfants russes en émigration, alors [...] ces enfants, dans leur grande majorité, cesseront d'exister comme Russes, ils s'intégreront inéluctablement dans les milieux étrangers ${ }^{41}$,

soulignait l'un des organisateurs de cette fête initiée par le Bureau pédagogique, le Zemgor, l'Union des villes et le groupe académique. Les bénéfices de cette manifestation étaient d'abord attribués aux lycées, puis aux multiples initiatives parascolaires destinées aux enfants scolarisés dans les écoles nationales (organisations de scouts, cours hebdomadaires de russe, écoles du dimanche, etc.).

Jusqu'à la Seconde Guerre mondiale, les Journées de la culture russe et de l'enfant se déroulèrent dans pratiquement tous les pays de la diaspora, y compris en Chine et aux États-Unis. La Journée de l'enfance est toujours célébrée à San Francisco $^{42}$. D'autres initiatives méritent d'être mentionnées, telle l'organisation du Foyer des amis de la culture russe par S. Štern ou encore la création à Paris en décembre 1926 du théâtre «Detskaja lira » sous la direction de l'artiste V. TsappKanevskij et de la ballerine A. Smolenska.

Fait assez remarquable, le soutien apporté à l'éducation des enfants rassembla des cercles d'horizons très divers. Ainsi le Comité de tutelle des enfants russes créé en France sous la présidence du diplomate et représentant du Conseil des ambassadeurs, M. N. Giers, était animé par des responsables paroissiaux, des représentants de la Croix-Rouge russe, du Zemgor, de l'Union des officiers russes, du groupe académique, etc. Vingt organisations étaient ainsi représentées dans ce comité, soulignant s'il en était besoin, l'importante mobilisation des différents acteurs de l'émigration en faveur de l'éducation des jeunes générations.

40. GARF, f. R 5785, op. 1, d. 10,1. 146.

41. GARF, f. R 5851, op. 1, d. 7,1. 82.

42. En 2004, il s'agissait de la $73^{\circ}$ édition de cette manifestation. 
En conclusion, et mieux que des données statistiques sur les résultats de l'action éducative, ce sont les témoignages des enfants eux-mêmes qui peuvent permettre d'apprécier l'œuvre entreprise. Suite à l'initiative prise en 1923-1924 de recueillir leurs souvenirs depuis l'année 1917, beaucoup d'élèves des écoles russes firent part du caractère, pour eux, salvateur de ces établissements. Une élève du lycée de Třebova écrivait ainsi : «En arrivant à Constantinople, je suis tout de suite entrée au lycée et là a commencé une nouvelle vie, pleine d'espoir et de rêves. Tout le passé n'était qu'un affreux cauchemar ${ }^{43}$. Un autre élève du lycée de Chumen concluait ses mémoires en affirmant : «Maintenant, je sens que le lycée m'a donné beaucoup, beaucoup. Et je remercie chaleureusement et je remercierai toujours nos chers enseignants $»^{44}$. Les citations pourraient être multipliées, mais toutes témoignent de la pleine conscience des élèves face à l'immense effort consenti par les acteurs sociaux de l'exil pour transmettre l'héritage culturel et éducatif russes à ceux qui n'avaient surtout connu de leur pays qu'une terre à feu et à sang.

\section{GARFMoscou}

garf@online.ru 